\title{
Cytocompatibility of Plasma and Thermally Treated Biopolymers
}

\author{
Petr Slepička, ${ }^{1}$ Iva Michaljaničová, ${ }^{1}$ Nikola Slepičková Kasálková, \\ Petr Sajdl, ${ }^{2}$ Zdeňka Kolská, ${ }^{3}$ and Václav Švorčík ${ }^{1}$ \\ ${ }^{1}$ Department of Solid State Engineering, Institute of Chemical Technology, 16628 Prague, Czech Republic \\ ${ }^{2}$ Department of Power Engineering, Institute of Chemical Technology, 16628 Prague, Czech Republic \\ ${ }^{3}$ Faculty of Science, J. E. Purkynè University, 40096 Ústí nad Labem, Czech Republic \\ Correspondence should be addressed to Petr Slepička; petr.slepicka@vscht.cz
}

Received 3 June 2013; Revised 21 June 2013; Accepted 21 June 2013

Academic Editor: Jiamin Wu

Copyright (C) 2013 Petr Slepička et al. This is an open access article distributed under the Creative Commons Attribution License, which permits unrestricted use, distribution, and reproduction in any medium, provided the original work is properly cited.

\begin{abstract}
This paper is focused on the surface characterization of plasma and consequently thermally treated biocompatible polymers. PLLA (poly(L-lactide acid) and PMP (poly-4-methyl-1-pentene) are studied. The influence of Ar plasma treatment on the surface polarity of substrate measured immediately after treatment and during the polymer surface aging is studied. Surface roughness, morphology, wettability, and surface chemistry were determined. Plasma treatment leads to significant changes in PLLA surface morphology and chemistry, with the PMP being slightly affected. The higher resistance to plasma fluence results in smaller ablation of PMP than that of PLLA. The plasma treatment improves cell adhesion and proliferation on the PMP. Plasma treatment of PLLA influences mostly the homogeneity of adhered and proliferated VSMC.
\end{abstract}

\section{Introduction}

The aim of the material engineering is to observe and modify the properties of materials with the purpose of consecutive application in different branches of science and engineering. The interdisciplinary studies are arising from the fields of applied physics and chemistry, which are focusing on the relationships between materials' structure on the molecular and atomic levels and their macroscopic behavior. The nanotechnology allows for manipulating and studying the materials on an atomic level (or the properties less than $100 \mathrm{~nm}$ in one dimension), so that both the manipulation and sensitive analysis can be performed [1]. The nanotechnology involves the study of metals, semiconductors, special glasses, and also polymers in different forms, which can be also applied as biomaterials.

The observations in this field are crucial for the applications in medicine, pharmacology, biology, tissue engineering [2], or material science. The usage of biomaterials (polymers, ceramics, and others) can be found in dental replacements
[3], orthopedic or spinal implants [4], or the materials for the application of targeted drug release [5]. The biomaterials can be either separate types, for example, metals, ceramics or polymers, or their combinations of specific type, composites, which are combined from one or more substances with different properties. The biocompatibility of polymeric materials can be also increased by excimer lamp irradiation [6] ion beam modification [7] or consequent carbon layer deposition [8]. Such materials can be successfully applied in tissue engineering $[9,10]$.

Polymers exhibit interesting mechanical and physicochemical properties as a scaffold in tissue engineering [11]. The surface physicochemical properties of polymer cell carriers can be also improved by surface grafting processes [12-14], which can mainly enhance the cell adhesion and proliferation. The methods of modification allow significant changes of surface properties, while the bulk properties (mostly mechanical) remain almost unchanged. Application of biomaterials for the cell cultivation is determined by the cell adhesion and their consecutive cell growth. Cell adhesion 
or their tenacity is closely connected with material surface properties, for example, its wettability, polarity, surface energy, electrical properties, morphology, roughness, and chemical structure. These properties can be also naturally altered by various types of surface modification [15-17]. Wettability (water contact angle) is one of the most important parameters influencing the cell's adhesion. The contact angle measurement is mostly used for its quantitative analysis. There is no "optimal" contact angle for the cell adhesion and proliferation in common; different cell types "need," for example, different surface polarity and surface roughness. Neither too hydrophilic nor hydrophobic materials are demanded [18]. For example, the observation of adhesion and growth of Chinese hamster ovary $(\mathrm{CHO})$ cells revealed that the best results were obtained for the surface with contact angle of $50^{\circ}$ [19]. The amount of "well" adhered cells can be also influenced by the chemical structure, that is, types of functional group present on the polymer surface and their concentration.

This work studies surface properties of two materials: (i) poly(L-lactide acid) (PLLA) and (ii) poly-4-methyl-1pentene (PMP). The PLLA substrate was chosen since it is biodegradable polymer; the biodegradation after successful growth of different types of cell lines is its great benefit. PMP is widely used polymer in tissue engineering with excellent mechanical properties. Therefore the improvement of its biocompatibility and the altering of its surface properties are of great importance. The initial part is devoted to the influence of modification of Ar plasma on polarity immediately after modification and during aging process. The thickness of ablated layer and surface morphology induced by plasma was determined. In the next section, attention is paid to thermal stress in combination with plasma adjustment, during which the study was focused on the surface morphology. The results from adhesion and proliferation of cells on selected substrates are also presented.

\section{Materials and Methods}

2.1. Materials, Plasma, and Heat Treatment. Biopolymer (i) poly(L-lactide acid) (PLLA, density $1.25 \mathrm{~g} \mathrm{~cm}^{-3}, T_{q}=60^{\circ} \mathrm{C}$, crystallinity $60-70 \%, 50 \mu \mathrm{m}$ thick foils, supplied by Goodfellow, Ltd.) and (ii) linear isotactic poly-4-methyl-1-pentene (PMP, density $0.835 \mathrm{~g} \mathrm{~cm}^{-3}, T_{g}=25^{\circ} \mathrm{C}, T_{m}=228^{\circ} \mathrm{C}$, crystallinity $52 \%, 50 \mu \mathrm{m}$ thick foils, supplied by Goodfellow, Ltd.) were used for the experiments.

The samples were modified in diode plasma discharge on Balzers SCD 050 device for 0-480 s, using DC Ar plasma (gas purity was 99.997\%, power 5 and $10 \mathrm{~W}$ ). Chamber parameters were Ar flow $0.31 \mathrm{~s}^{-1}$, Ar pressure $10 \mathrm{~Pa}$, electrode area $48 \mathrm{~cm}^{2}$, the interelectrode electrode distance of $50 \mathrm{~mm}$, and chamber volume $1000 \mathrm{~cm}^{3}$.

Thermal treatment of the polymers was accomplished in thermostat BINDER. The samples were heated at $60^{\circ} \mathrm{C}$ (PLLA) and $160^{\circ} \mathrm{C}$ (PMP, Vicat softening point). The pristine and modified samples (immediately after plasma treatment) were heated for 30 minutes and then they were cooled down to room temperature.

\subsection{Measurement Techniques}

2.2.1. Contact Angle and Surface Free Energy. Contact angle was determined by goniometry with static water drop method. The measurements of water contact angles (error $\pm 5 \%$ ) were performed using distilled water ( 9 different positions) using the Surface Energy Evaluation System (SEE System, Advex Instruments, Czech Republic). By Automatic pipette the water drop of volume $(8.0 \pm 0.2) \mathrm{mL}$ was deposited on the polymer's surface and the consequent photo was evaluated. Estimation of surface energy was also based on a measurement with SEE System, and two liquids (water and glycerol) were used. On the basis of Owens-Wendt method the values of surface free energy for the two liquids were evaluated. The measurement was carried out at room temperature.

2.2.2. X-Ray Photoelectron Spectroscopy. The presence of oxygen and carbon in the modified PMP and PLLA surface layer was proved by X-ray photoelectron spectroscopy (XPS). An Omicron Nanotechnology ESCAProbeP spectrometer was used. The exposed and analyzed area had a dimension of $2 \times 3 \mathrm{~mm}^{2}$. The X-ray source was monochromated at $1486.7 \mathrm{eV}$ and the measurement was performed with a step size of $0.05 \mathrm{eV}$. Characteristic $\mathrm{O}(1 \mathrm{~s})$ and $\mathrm{C}(1 \mathrm{~s}) \mathrm{S}(2 \mathrm{~s})$ peaks were searched for. Atomic concentrations of elements were determined by CASA XPS program using integrated area of spectrum lines and relative sensitivity factors which are quoted in the database of CASA XPS. The intensity calibration was carried out by the measurement of copper and the calculation of calibration constant for each used pass energy.

2.2.3. Surface Morphology. Surface morphology and roughness of the pristine and modified polymer samples were examined by the AFM technique using a VEECO CP II device in tapping mode. A Si probe RTESPA-CP with the spring constant $20-80 \mathrm{~N} \mathrm{~m}^{-1}$ was used. The mean roughness value $\left(R_{a}\right)$ represents the arithmetic average of the deviations from the central plane of the sample.

2.2.4. Gravimetry. Thickness of the ablated surface layer after plasma treatment was measured using a Mettler Toledo UMX2. In order to enhance the sensitivity of the measurement the samples (diameter $2.5 \mathrm{~cm}$ ) were exposed to plasma from both sides. The thickness of the ablated layer was calculated from the change in weight of 10 samples before and after the treatment using tabulated polymer density. The depolarization high-frequency gate was used to eliminate surface discharge in order to minimize the influence of surface electrostatic charge on the measurement.

2.2.5. Electrokinetic Analysis. Zeta potential determination of all samples was accomplished on SurPASS Instrument (Anton Paar GmbH, Austria) by two methods (streaming current and streaming potential) and calculated by two equations (Helmholtz-Smoluchowski, HS, and FairbrotherMastins, FM). Samples were studied inside the adjustable gap cell with an electrolyte of $0.001 \mathrm{~mol} \mathrm{dm}^{-3} \mathrm{KCl}$ at constant 
$\mathrm{pH}=6.1$ and at room temperature. Two samples of each surface were measured four times with the relative error of $5 \%$.

\subsection{Cell Culture, Adhesion, Proliferation, and Cell Number} Determination. For cell culture experiments, four pristine and the same number of plasma modified samples (power 5 and $10 \mathrm{~W}$, exposure times 40 and $240 \mathrm{~s}$ ) were used. The samples were sterilized for 1 hour in ethanol (75\%), air-dried, inserted into polystyrene 12-well plates (TPP, Switzerland), and seeded with vascular smooth muscle cells (VSMCs) derived from the rat aorta by an explantation method. VSMCs were seeded on the samples with the density of 50.000 cells/well (i.e., about 17.000 cells cm${ }^{-2}$ ) into $3 \mathrm{~mL}$ of Dulbecco's modified Eagle's Minimum Essential Medium (DMEM; Sigma, USA, Cat. no. D5648), containing 10\% fetal bovine serum (FBS; Sebak GmbH, Aidenbach, Germany). Cells were cultivated at $37^{\circ} \mathrm{C}$ in a humidified air atmosphere containing $5 \%$ of $\mathrm{CO}_{2}$. The number and the morphology of initially adhered cells were evaluated 24 hours after seeding. The cell adhesion and proliferation activity was estimated from the increase in the cell numbers achieved 1,2, 5, and 7 days after seeding. Phosphate buffer (PBS) was used for cell rinsing, and trypsine with ethylenediaminetetraacetic acid (EDTA) was used for cell releasing from the substrate surface.

For the cell number determination the method of visual field was chosen. The results obtained on pristine and plasma treated PLLA and PMP were confronted with those obtained on TCPS (tissue polystyrene). The method was used for the cell number determination of adhered cells ( 24 and 48 hours from seeding) and the number of proliferated cells (120 and 168 hours from seeding). The samples were rinsed with PBS and fixed by $70 \%$ ethanol solution $\left(-20^{\circ} \mathrm{C}\right)$. The fluorescence dyes were consequently used for the coloring of plasmatic membranes (Texas Red C2 maleimide) and chromosomes in cell nuclei (Hoechst \# 33342). The coloring was performed for 1 hour at room temperature. When colored the samples were rinsed with PBS again and placed between microscopic glasses. The Olympus IX51 was used for taking 20 photos from different positions on each of the samples. The number of cells was consequently processed with Lucia software and Origin 8.0. The Vi-CELL utilizes the widely accepted trypan blue dye exclusion method to determine cellular viability. When cells die their membranes become permeable, thus allowing for the uptake of the trypan blue dye. As a result, the dead or nonviable cells become darker than the viable cells. It is this contrast that is measured to determine viability.

The standard deviations for the data introduced in the graphs in the paper were calculated with the standard Excel function STDEV (corrected sample standard deviation):

$$
s=\sqrt{\frac{1}{N-1} \sum_{i=1}^{N}\left(x_{i}-\bar{x}\right)^{2}},
$$

where $\left(x_{i}, \ldots, x_{n}\right)$ are the observed sample values and $\bar{x}$ is the mean value of these observations, while the denominator $N$ stands for the number of the values.
The $R_{a}$ determination (average roughness, $\mathrm{AFM}$ ) is constructed from the matrix $512 \times 512$ points; the equation is

$$
R_{a}=\frac{\sum_{i=1}^{N}\left|Z_{i}-Z_{\mathrm{cp}}\right|}{N}
$$

where $Z_{\mathrm{cp}}$ is the value of the central plane, $Z_{i}$ is the actual $Z$ value, and $N$ is the number of points, where the $Z_{i}$ is evaluated.

\section{Results and Discussion}

3.1. Contact Angle and Surface Energy Measurement. The influence of plasma treatment (exposure time 5-240 s, power 5 and $10 \mathrm{~W}$ ) on PLLA and PMP surface polarity was studied with goniometry which can be determined by contact angle measurement [20-23]. From contact angles of distilled water and glycerol the surface energy was observed. As the results indicate, the pristine samples exhibit low values of surface energy and the plasma treatment causes its significant increase (Figures 1(a) and 1(b)). The value of surface energy $31.9 \mathrm{~mJ} \mathrm{~m}^{-2}$ was determined for pristine PLLA. Even the short time of plasma treatment results in sharp increase in polymer surface energy. The PMP samples exhibit "more regular" behavior. The value of pristine PMP sample was determined to have surface energy $18.8 \mathrm{~mJ} \mathrm{~m}^{-2}$. By the plasma treatment the value of the PMP's surface energy significantly grows. For higher exposure times $(>15 \mathrm{~s})$ the energy remains almost constant with the value of $74 \mathrm{~mJ} \mathrm{~m}^{-2}$.

3.2. Aging of Treated Polymers. The good stability of substrate properties in tissue engineering is important for their application as biomaterials. Due to this we study also the behavior of properties during aging of samples. For potential application of biomaterial in tissue engineering it is very important to determine whether the material exhibits "constant" behavior in time. It is clear from Figure 2 that the time from the plasma treatment significantly influences the values of contact angle. The aging measurements were performed for power 5 and $10 \mathrm{~W}$ and exposure time $240 \mathrm{~s}$. This combination resulted previously in the highest decrease in contact angle both for PLLA and PMP; therefore the combination of parameters was chosen for the aging studies. Both the tested polymers exhibit the same trend of contact angle during aging process in general. The PLLA sample exhibits the value of pristine foil $71.1^{\circ}$, as was mentioned before. The sharp decrease immediately after the treatment is changed to increase with increasing aging time. This increase reaches the saturated value of $83^{\circ}$ for PLLA treated with $5 \mathrm{~W}$ (Figure 2(a)), with the aging time being 50 hours. Also the treatment of PLLA with $10 \mathrm{~W}$ leads after 50 hours of aging process to estimation of a saturated value of ca $87^{\circ}$ (Figure 2(a)). Both saturated values are higher than pristine PLLA sample, which is the result of a lower polarity of PLLA after the aging process. A significant difference is that the PMP samples also exhibits almost similar values for both applied plasma powers (Figure 2(b)), with the value being lower than that for pristine PMP (ca $104^{\circ}$ ). The orientation of the oxygen containing groups, the rearrangement of degraded macromolecules and molecular 


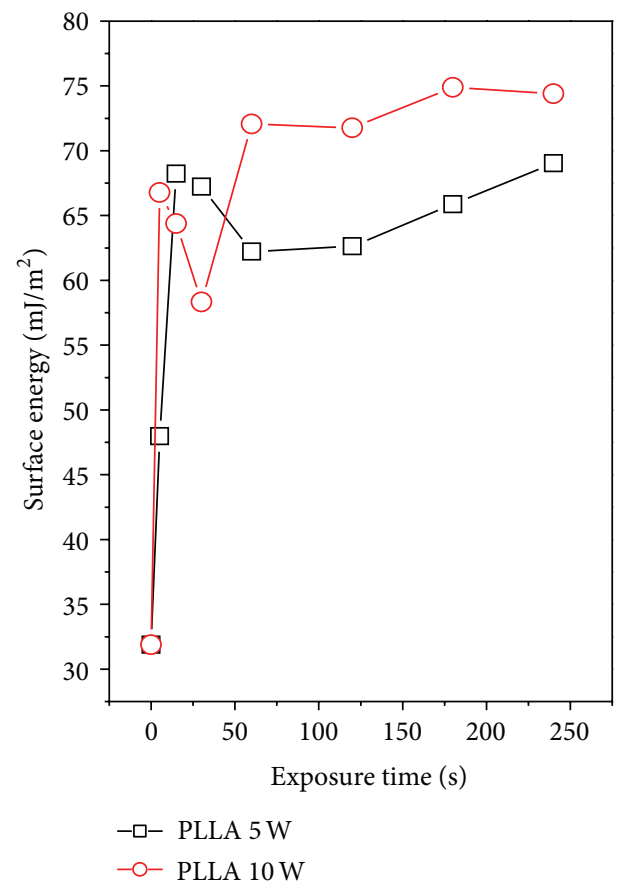

(a)

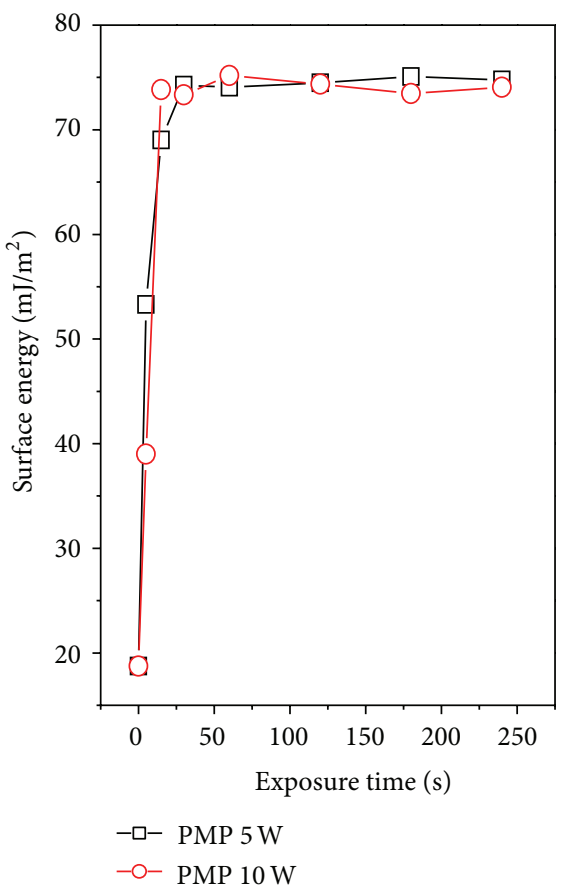

(b)

Figure 1: Dependence of the surface energy of plasma treated (with 5 and $10 \mathrm{~W}$ ) PLLA (a) and PMP (b) on Ar plasma exposure time.

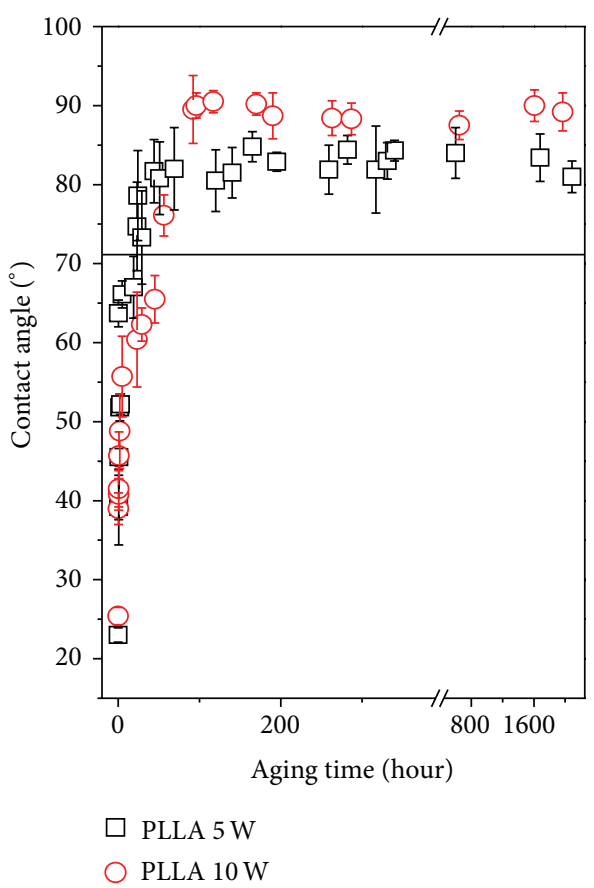

(a)

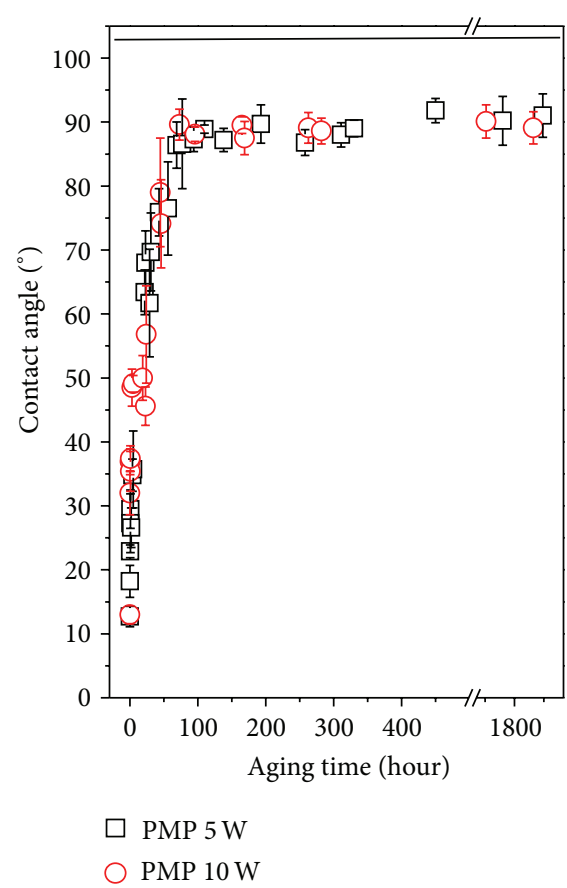

(b)

FIGURE 2: Dependence of contact angle of plasma treated (with 5 and $10 \mathrm{~W}$ ) PLLA (a) and PMP (b) on aging time. Values for pristine polymers are shown by solid line. 


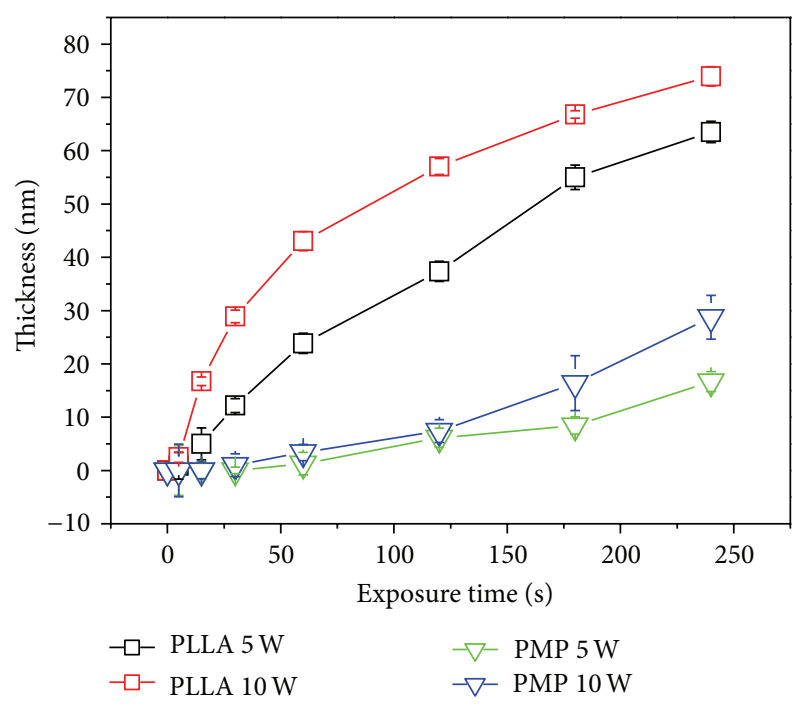

Figure 3: Dependence of the thickness of the ablated layer of PLLA and PMP on the Ar plasma exposure time (power 5 and $10 \mathrm{~W}$ ).

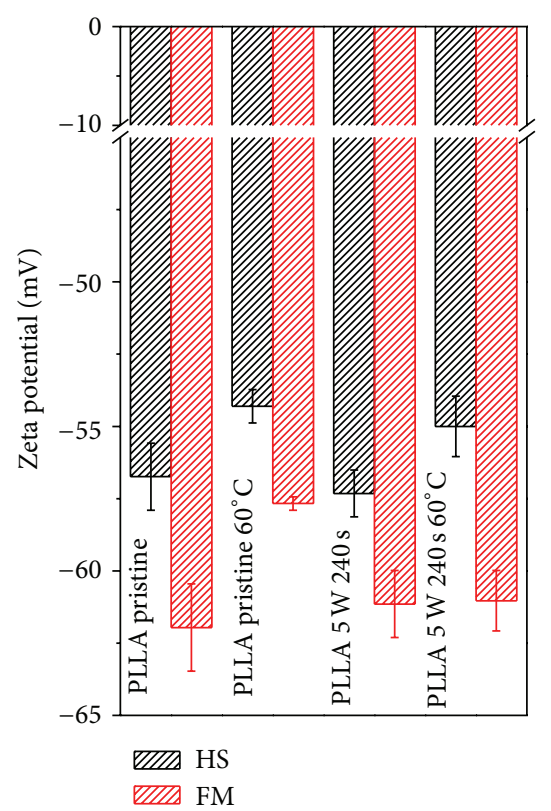

Figure 4: Zeta potential results for PLLA pristine (PLLA), plasma treated (PLLA $/ 5 \mathrm{~W} / 240 \mathrm{~s})$, and annealed ones at $60^{\circ} \mathrm{C}\left(\mathrm{PLLA} / 60^{\circ} \mathrm{C}\right.$ and PLLA $\left./ 5 \mathrm{~W} / 240 \mathrm{~s} / 60^{\circ} \mathrm{C}\right)$. HS means the streaming current method and Helmholtz-Smoluchowski equation; FM means the streaming potential method and Fairbrother-Mastins equation.

fragments into the polymer bulk, which may occur on the polymer surface in the process of aging, is the main reason for the increase in contact angle during the aging process.

3.3. Ablation, Zeta Potential, and Surface Morphology. During plasma treatment process the ablation of polymer takes place. The ablation has been studied gravimetrically. The ablation loss of polymer was consecutively recalculated to polymer thickness. From Figure 3 it is apparent that the exposure time and power significantly influence the ablation of both polymers. The samples were modified using plasma by 5 and $10 \mathrm{~W}$ and exposure times from 5 to $240 \mathrm{~s}$. As expected, with increasing the exposure power and time the higher ablation loss is apparent. This phenomenon is more progressed in case of PLLA. This implicates that surface of PMP is more resistant to plasma treatment than PLLA surface. The highest ablation loss was observed for PLLA foil modified with $10 \mathrm{~W}$ and $240 \mathrm{~s}$. The thickness of ablated material was ca $73 \mathrm{~nm}$ (see Figure 3). The mass loss for treatment parameters ( $10 \mathrm{~W}$ and $240 \mathrm{~s}$ ) was $18.5 \mu \mathrm{g} \mathrm{cm}^{-2}$ for PLLA and $4.8 \mu \mathrm{g} \mathrm{cm}^{-2}$ for PMP. From Figure 3 it can be concluded that the ablation is more pronounced on the PLLA.

Results of electrokinetic analysis are presented in Figure 4. Because PMP was the slightly affected in comparison with PLLA, we present results for only PLLA. The trend of zeta potential changes obtained by both of the applied methods, streaming current (HS) and streaming potential (FM), is the same. As it is clear, the plasma treatment (after the aging process and surface relaxation) does not exhibit significant changes in surface charge represented by zeta potential. It can be explained by the high ablation of surface during treatment discussed above. On the other hand, zeta potential of pristine PLLA and also plasma treated one increases after annealing at $60^{\circ} \mathrm{C}$ due to increasing surface polarity. Therefore it can be concluded that the annealing process has a significant effect on the ability to influence the surface-ion interaction.

Surface morphology of pristine and treated samples was studied with AFM. The pristine PLLA exhibits the surface morphology with no obvious surface irregularities and surface roughness $R_{a}=6.9 \mathrm{~nm}$ (Figure 5). The plasma treatment has significant effect on the surface morphology. As a result of the plasma irradiation, the surface roughness dramatically increases and sharp fragments appear on the modified surface. The ablation takes place (as discussed in Section 3.3, 1st paragraph), preferentially with the amorphous phase being ablated and the crystalline amount of PLLA surface being revealed. Rather different situation arises in case of PMP. A mild decrease in surface roughness after treatment of PMP surface has been observed. The surface morphology is almost unaltered by the plasma treatment. From the surface morphology study it can be concluded that PMP is more plasma resistant than PLLA and no significant changes are made as a result of plasma treatment. The surface morphology can be also significantly influenced by the surface etching, which will be discussed later.

3.4. Thermal Treatment. The thermal treatment of pristine PLLA caused mild increase in surface roughness (see Figures 5 and 6). The change of surface morphology of pristine PLLA induced by thermal treatment is minor. The situation is dramatically changed after plasma treatment. It is obvious from Figure 5 that the combination of plasma and consecutive thermal treatment leads to significant changes in surface morphology and roughness. This change is strongly influenced by the plasma power. It is evident that lower plasma power 


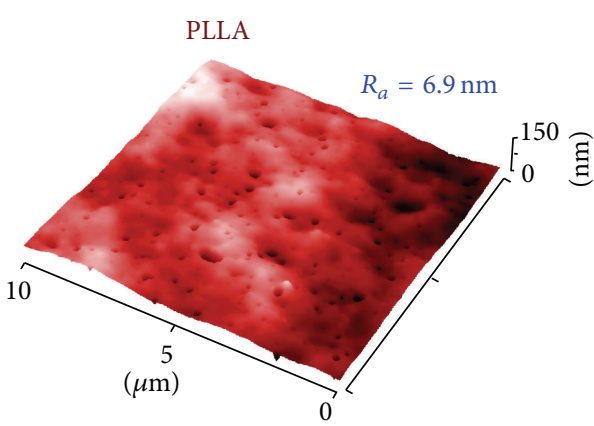

(a)

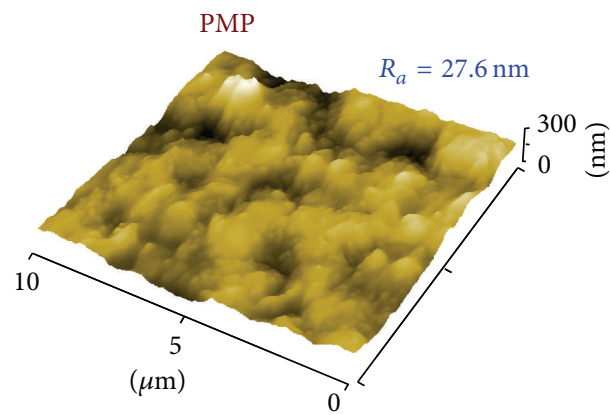

(c)

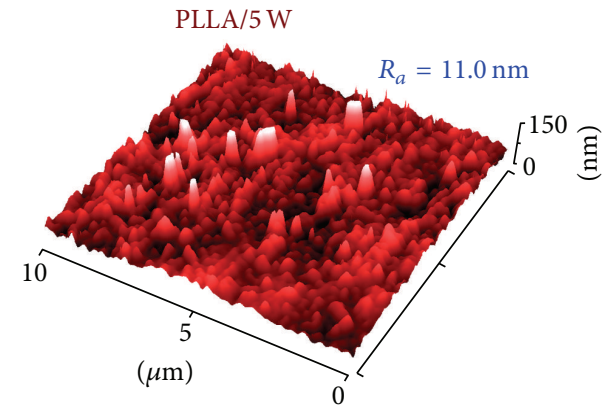

(b)

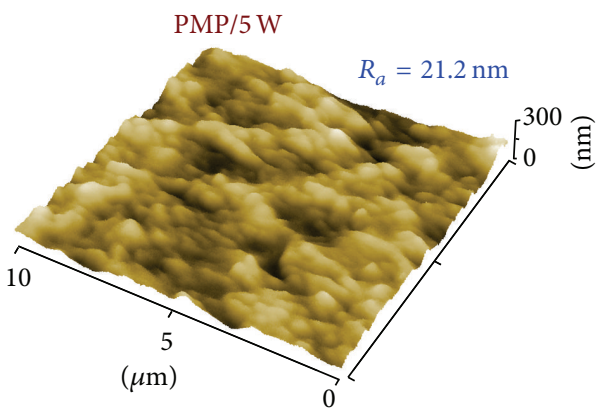

(d)

FIGURE 5: AFM images of pristine polymers (PLLA and PMP) and polymers treated with Ar plasma (power $5 \mathrm{~W}$ and time $240 \mathrm{~s}$ ) (PLLA/5 W and $\mathrm{PMP} / 5 \mathrm{~W}) . R_{a}$ is average surface roughness in $\mathrm{nm}$.

(5 W) in combination with thermal treatment leads to change of surface roughness from 7.0 to $13.0 \mathrm{~nm}$ and the surface structure gets a "worm-like character." The most pronounced changes were observed for the samples modified for $10 \mathrm{~W}$ (240 s) and then thermally treated. The dramatic increase in surface roughness up to $23 \mathrm{~nm}$ combined with significant change of surface morphology (worm-like structure) was observed. The changes in surface morphology and roughness are probably induced by different amount of crystalline and amorphous phase on the very surface of modified PLLA layer, affecting the melting process and process of solidification.

The PMP foils were heated to $160^{\circ} \mathrm{C}$ (Vicat softening point) and their morphological stability was determined. For the sake of clarity only the surface morphology of PLLA heated samples was introduced in Figure 6. The thermal treatment has no significant effect neither on surface morphology nor roughness in case of plasma modified PMP samples. After thermal treatment, both the surface morphology of 5 and that of $10 \mathrm{~W}$ exposed PMP samples remained only mildly altered. This effect is in good correspondence with previously obtained results from gravimetry (see Figure 3). The higher resistance to plasma influence results in smaller ablation of PMP material and thus supports the morphological stability during thermal treatment of plasma exposed samples.

3.5. Chemistry of Modified Polymers. The surface chemical structure of polymers was studied with XPS. The selected results for PMP and PLLA treated with 5 and $10 \mathrm{~W}$ are introduced in Table 1 . The oxygen concentration dramatically increases on the PMP surface after plasma treatment (from 0.3 to $20.5 \%$ ). The oxygen concentration is further increased by the surface aging. This surprising fact can be probably caused by additional surface oxidation and polymer segments reorientation (increase of $4 \%$ ). The difference may be due to combination of applied plasma power, which together with surface morphology changes, and air oxygen could induce this slight increase in surface oxygen concentration. The observation of increasing oxygen concentration during aging process was also observed for PMMA [24].

3.6. Cells Adhesion and Proliferation. For the cell proliferation and differentiation studying their adhesion on the substrate is essential. After successful adhesion the lag phase occurs, usually between the first and the second day from seeding, but it can be up to 72 hours. The cells adapt on their new environment. After this phase the proliferation phase develops. For the cell compatibility studies the PLLA was selected. The vascular smooth muscle cells (VSMC) were seeded also on tissue polystyrene (TCPS) as a material standard (Figure 7).

The cell adhesion represents the first stage of the cellsubstrate interaction and the quality of this phase influences the cell ability to proliferate and differentiate in the contact with the substrate. The initial number of cells $\left(17000 \mathrm{~cm}^{-2}\right)$ was seeded on studied substrates and the decrease to 4500 cells $\mathrm{cm}^{-2}$ after 24 hours was observed. This decrease was caused by the processes of adhesion of cell to substrate, where approximately one fourth of the cells survived. If we 


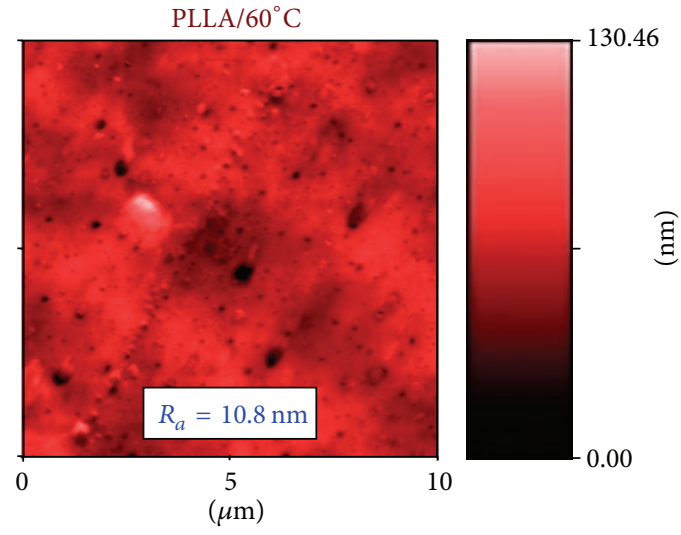

(a)

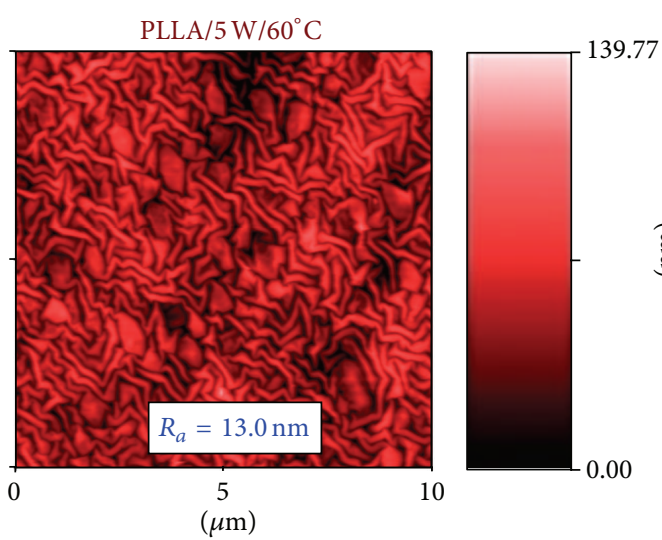

(b)

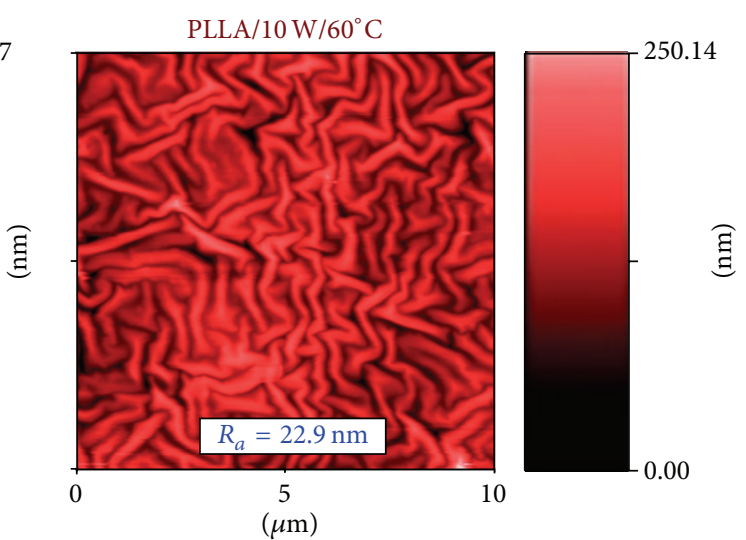

(c)

Figure 6: $2 \mathrm{D}$ AFM images of PLLA thermally treated by $60^{\circ} \mathrm{C}$ : pristine (PLLA $/ 60^{\circ} \mathrm{C}$ ), PLLA treated with plasma ( $5 \mathrm{~W}$ and $240 \mathrm{~s}$ ) $\left(\mathrm{PLLA} / 5 \mathrm{~W} / 60^{\circ} \mathrm{C}\right)$, and PLLA treated with plasma $(10 \mathrm{~W}$ and $240 \mathrm{~s})\left(\mathrm{PLLA} / 10 \mathrm{~W} / 60^{\circ} \mathrm{C}\right) . R_{a}$ is the surface roughness in $\mathrm{nm}$.

compare the shape and distribution of cells during the initial adhesion stage we can conclude that the cells of pristine polymers are small, round shaped, and not well spread. The TCPS exhibits slightly better distribution of cells on the surface. The modified surfaces (both PLLA and PMP) exhibit during the initial adhesion stage (first day) the best results according to the shape and distribution over the surface. The Live/Dead assay by the Vi-CELL instrument (Table 2) revealed that the results except for pristine PMP (first day) were better than $70 \%$ and for TCPS were better than $80 \%$.

48 hours after seeding the evident increase in cell number was detected (about 2000 cells $\mathrm{cm}^{-2}$ ) for the samples modified with plasma (5 W, 40 and $240 \mathrm{~s}$ ) and (10 W, $240 \mathrm{~s})$ as well as on standard TCPS. The pristine PLLA and PLLA modified ( $10 \mathrm{~W}, 40 \mathrm{~s})$ exhibited almost no progress in cell number. After 5 days from seeding, when the lag phase is over, the cell differentiation starts to increase. The most successful was evaluated PLLA sample modified with $10 \mathrm{~W}$ and $240 \mathrm{~s}$ after 5 days from seeding (Figure 7). The lowest cell number was detected, on the contrary, on the PLLA modified with $5 \mathrm{~W}$ and $240 \mathrm{~s}$. The PLLA polymer itself also exhibited "very good" results, that is, high cell numbers. The best result for PMP substrate was determined for modification with
$5 \mathrm{~W}$ and $40 \mathrm{~s}$ (Figure 7). The reason for the enhancing of VSMC proliferation on PLLA with $10 \mathrm{~W}$ and $240 \mathrm{~s}$ and PMP with $5 \mathrm{~W}$ and $40 \mathrm{~s}$, compared to pristine samples, lies in the change of its roughness in combination with theirs surface wettability. Compared to the surface wettability, roughness, and chemistry the PLLA modified with $10 \mathrm{~W}$ and $240 \mathrm{~s}$ has the pronounced globular structures on its surface with high roughness, while the PMP modified with $5 \mathrm{~W}$ and $40 \mathrm{~s}$ exhibits significant increase in oxygen concentration compared to pristine PMP, which may be the main reason for the enhancing of VSMC proliferation.

The selected pictures of PLLA polymer modified with $10 \mathrm{~W}$ for 40 and $240 \mathrm{~s}$ are introduced in Figure 8 . The photos from comparative material TCPS after the first and seventh days are introduced in Figures $8(\mathrm{a})$ and $8(\mathrm{~b})$ too. The results on TCPS revealed the highest number of proliferated cells from all studied samples. The inhomogeneous cell distribution in the first stage of the cell growth is apparent (Figures $8(\mathrm{c})$, and $8(\mathrm{~d}))$. The dimensions of cells after successful adhesion process may be in relatively wide interval. Also the shape of the cells implicates that the "width" dimension could be approx. 40-50 $\mu \mathrm{m}$ and the "length" dimension about $150-200 \mu \mathrm{m}$. The diameter of cell core is approx. $15 \mu \mathrm{m}$. The 


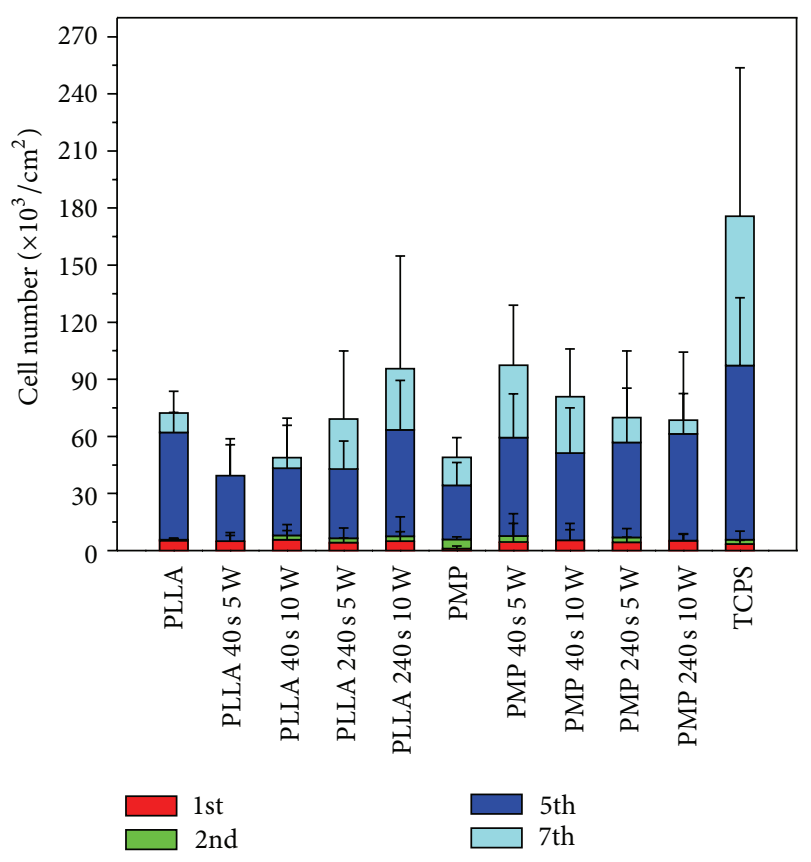

FIGURE 7: Dependence of the number of adhered and proliferated cells after 1, 2, 5, and 7 days from seeding on pristine PLLA and PMP and PLLA and PMP treated in plasma (power 5 and $10 \mathrm{~W}$, time 40 and $240 \mathrm{~s}$ ). The values for tissue polystyrene as standard material (TCPS) were also introduced for comparison.

dimensions of the cell significantly depend on the density of cells on particular surface area. Seven days from seeding the cells are joined into conglomerates and form continuous layer (Figure $8(\mathrm{f})$ ). From those figures it is obvious that the higher number of cells was determined on the sample treated with power $10 \mathrm{~W}$ and $240 \mathrm{~s}$, but the cell's dimensions are significantly higher in comparison to those on TCPS (see Figure 8).

\section{Conclusions}

The influence of Ar plasma and thermal treatment on the surface properties of PLLA and PMP was determined by different techniques. The process of aging of the modified polymers was studied too. The pristine and treated polymers were used as substrates for cultivation of VSMC. The plasma treatment significantly alters the contact angle between surface and water (wettability) of polymer. Due to plasma treatment the contact angle decreases and the surface energy increases. With the increasing aging time the surface polarity is spontaneously reduced. The ablation caused by $\mathrm{Ar}$ plasma was more pronounced on the PLLA in comparison to PMP. The surface morphology of PLLA was significantly altered with both plasma modification and consecutive thermal treatment. The higher resistance to plasma treatment resulted in smaller ablation of PMP and thus supported the morphological stability during thermal treatment of plasma treated samples. The increase in oxygen concentration on PMP surface induced by plasma treatment was proved. The
TABLE 1: The element concentration on the PMP and PLLA samples. The values were determined by XPS method for pristine, plasma treated (5 W or $10 \mathrm{~W}, 240 \mathrm{~s}$ ), plasma treated, and aged samples.

\begin{tabular}{lcc}
\hline Sample & \multicolumn{2}{c}{ Element concentration (at. \%) } \\
Carbon & Oxygen \\
\hline PMP & & \\
$\quad$ Pristine & 99.7 & 0.3 \\
Plasma 5 W & 79.5 & 20.5 \\
Plasma 5 W/aged & 75.5 & 24.5 \\
Plasma 10 W & 80.3 & 19.7 \\
Plasma 10 W/aged & 75.6 & 24.4 \\
\hline PLLA & & \\
Pristine & 66.1 & 33.9 \\
Plasma 5 W & 66.3 & 33.7 \\
Plasma 5 W/aged & 61.5 & 38.5 \\
Plasma 10 W & 66.6 & 33.4 \\
Plasma 10 W/aged & 64.8 & 35.2 \\
\hline
\end{tabular}

TABLE 2: Live/Dead assay by the Vi-CELL instrument for the studied samples (in \%).

\begin{tabular}{lcccc}
\hline & 1st day & 2nd day & 5th day & 7th day \\
\hline PLLA 5 W 40 s & 87 & 83 & 81 & 79 \\
PLLA 10 W 40 s & 88 & 100 & 76 & 85 \\
PLLA 5 W 240 s & 73 & 76 & 76 & 83 \\
PLLA 10 W 240 s & 88 & 85 & 70 & 80 \\
PLLA pristine & 100 & 95 & 82 & 86 \\
\hline PMP 5 W 40 s & 88 & 87 & 83 & 82 \\
PMP 10 W 40 s & 88 & 88 & 84 & 82 \\
PMP 5 W 240 s & 100 & 92 & 78 & 82 \\
PMP 10 W 240 s & 92 & 93 & 81 & 79 \\
PMP pristine & 65 & 86 & 84 & 72 \\
\hline TCPS & 94 & 87 & 89 & 86 \\
\hline
\end{tabular}

plasma treatment improves cell adhesion and proliferation on the PMP. The higher number of cells was determined on the sample treated with power $10 \mathrm{~W}$ and $240 \mathrm{~s}$, but the cell's dimensions are significantly larger in comparison to those on TCPS. The potential application of plasma treated PLLA and PMP is connected with construction of biodegradable and biocompatible scaffolds for the growth of different types of cell lines.

\section{Conflict of Interests}

Petr Slepička, as the first author of the paper, hereby declares that he does not have any direct financial relation with the commercial identity mentioned in the paper that might lead to a conflict of interests for any of the authors. He hereby declares on behalf of all the coauthors of the paper that the same statement is valid for all of them. 


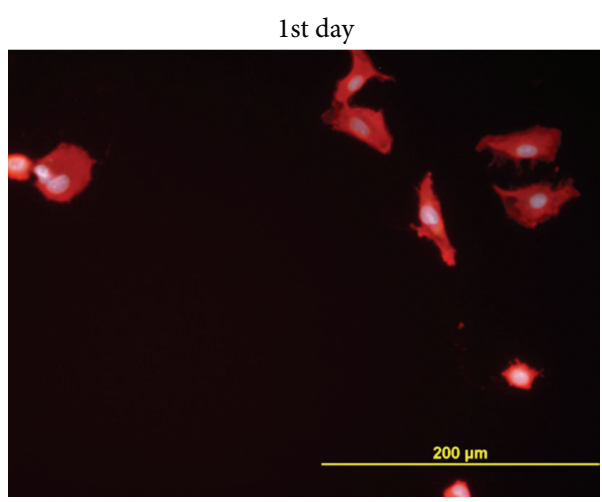

(a)

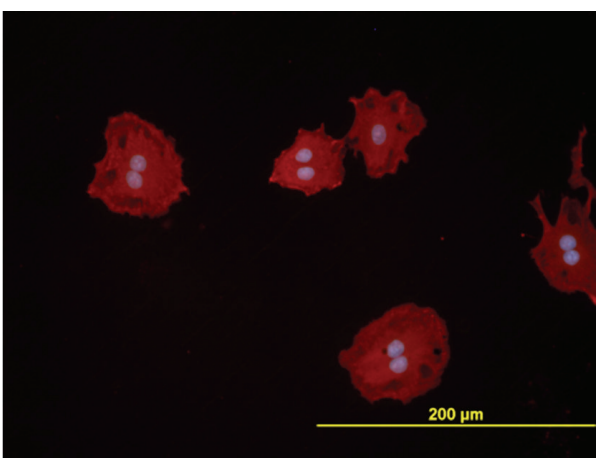

(c)

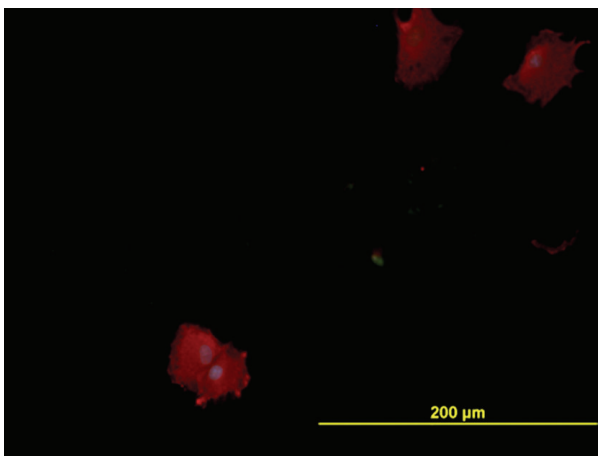

(e)

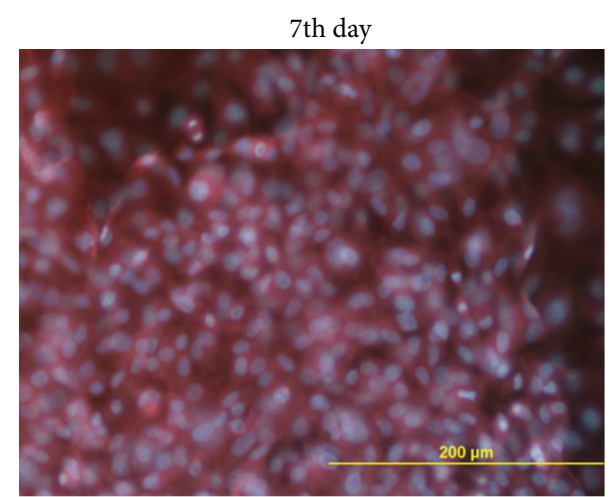

(b)

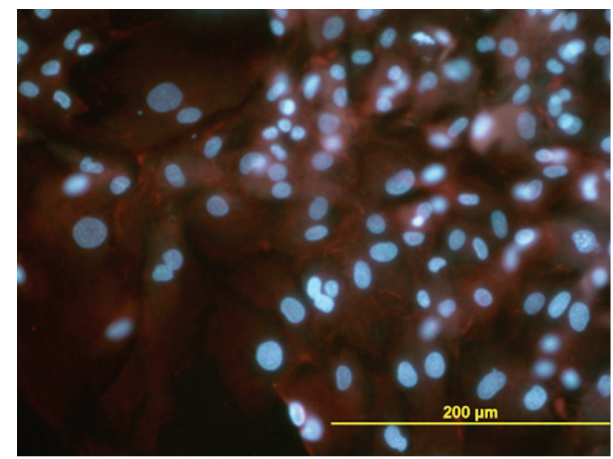

(d)

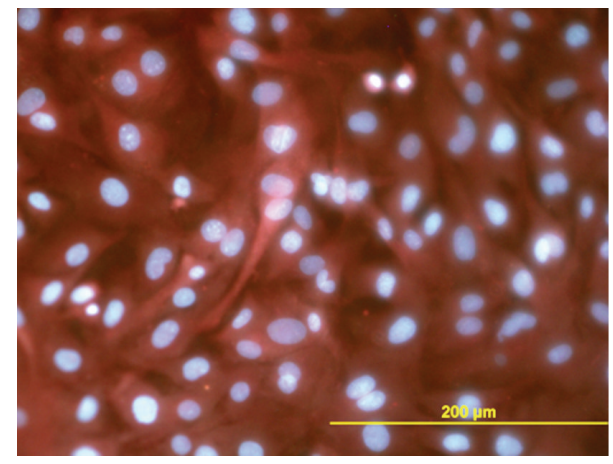

(f)

Figure 8: Photographs of adhered (first day, first column) and proliferated (seventh day, second column) VSMCs from seeding on tissue polystyrene (TCPS) ((a) and (b)), treated PLLA (10 W and $40 \mathrm{~s})((\mathrm{c})$ and (d)), and PLLA treated by $10 \mathrm{~W}$ and $240 \mathrm{~s}((\mathrm{e})$ and (f)).

\section{Acknowledgment}

This work was supported by the GACR under Project P108/ 12/G108.

\section{References}

[1] J. C. Miller, R. M. Serrato, J. M. Represas-Cardenas, and G. A. Kundahl, The Handbook of Nanotechnology, John Wiley \& Sons, Hoboken, NJ, USA, 2005.

[2] B. D. Ratner, Biomaterials Science: An Introduction to Materials in Medicine, Elsevier Academic Press, San Diego, Calif, USA, 2nd edition, 2004.

[3] N. Nakabayashi, "Dental biomaterials and the healing of dental tissue," Biomaterials, vol. 24, no. 13, pp. 2437-2439, 2003.
[4] S. M. Kurtz and J. N. Devine, "PEEK biomaterials in trauma, orthopedic, and spinal implants," Biomaterials, vol. 28, no. 32, pp. 4845-4869, 2007.

[5] M. L. Hans and A. M. Lowman, "Biodegradable nanoparticles for drug delivery and targeting," Current Opinion in Solid State and Materials Science, vol. 6, no. 4, pp. 319-327, 2002.

[6] J. Heitz, V. Švorčík, L. Bačáková et al., "Fibroblast and smooth muscle cell adhesion on UV-modified PTFE in ammonium atmosphera," Journal of Biomedical Materials Research A, vol. 67, no. 1, pp. 130-137, 2003.

[7] K. Walachová, V. Švorčík, L. Bačáková, and V. Hnatowicz, “Colonization of ion-modified polyethylene with vascular smooth muscle cells in vitro," Biomaterials, vol. 23, no. 14, pp. 29892996, 2002. 
[8] V. Švorčík, T. Hubáček, P. Slepička et al., "Characterization of carbon nanolayers flash evaporated on PET and PTFE," Carbon, vol. 47, no. 7, pp. 1770-1778, 2009.

[9] F. Groeber, M. Holeiter, M. Hampel, S. Hinderer, and K. Schenke-Layland, "Skin tissue engineering - in vivo and in vitro applications," Advanced Drug Delivery Reviews, vol. 63, no. 4-5, pp. 352-366, 2011.

[10] J. M. Kelm, V. Lorber, J. G. Snedeker et al., "A novel concept for scaffold-free vessel tissue engineering: self-assembly of microtissue building blocks," Journal of Biotechnology, vol. 148, no. 1, pp. 46-55, 2010.

[11] S. J. Hollister, "Porous scaffold design for tissue engineering," Nature Materials, vol. 4, no. 7, pp. 518-524, 2005.

[12] V. Švorčík, N. Kasálková, P. Slepička et al., "Cytocompatibility of Ar plasma-treated and Au nanoparticle-grafted PE," Nuclear Instruments and Methods in Physics Research B, vol. 267, no. 11, pp. 1904-1910, 2009.

[13] K. Ročková, V. Švorčík, L. Bačáková, B. Dvořánková, and J. Heitz, "Biocompatibility of ion beam modified and RGDgrafted polyethylene," Nuclear Instruments and Methods in Physics Research B, vol. 225, no. 3, pp. 275-282, 2004.

[14] H. Chen, L. Yuan, W. Song, Z. Wu, and D. Li, "Biocompatible polymer materials: role of protein-surface interactions," Progress in Polymer Science, vol. 33, no. 11, pp. 1059-1087, 2008.

[15] C. H. Wanke, J. L. Feijó, L. G. Barbosa, L. F. Campo, R. V. B. de Oliveira, and F. Horowitz, "Tuning of polypropylene wettability by plasma and polyhedral oligomeric silsesquioxane modifications," Polymer, vol. 52, no. 8, pp. 1797-1802, 2011.

[16] J. M. Goddard and J. H. Hotchkiss, "Polymer surface modification for the attachment of bioactive compounds," Progress in Polymer Science, vol. 32, no. 7, pp. 698-725, 2007.

[17] N. Kasálková, Z. Makajová, M. Pařízek et al., "Cell adhesion and proliferation on plasma-treated and poly(ethylene glycol)grafted polyethylene," Journal of Adhesion Science and Technology, vol. 24, no. 4, pp. 743-754, 2010.

[18] I. Pashkuleva, P. M. López-Pérez, H. S. Azevedo, and R. L. Reis, "Highly porous and interconnected starch-based scaffolds: production, characterization and surface modification," Materials Science and Engineering C, vol. 30, no. 7, pp. 981-989, 2010.

[19] J. H. Lee, J. W. Lee, G. Khang, and H. B. Lee, "Interaction of cells on chargeable functional group gradient surfaces," Biomaterials, vol. 18, no. 4, pp. 351-358, 1997.

[20] K. S. Kim, C. M. Ryu, C. S. Park, G. S. Sur, and C. E. Park, "Investigation of crystallinity effects on the surface of oxygen plasma treated low density polyethylene using X-ray photoelectron spectroscopy," Polymer, vol. 44, no. 20, pp. 62876295, 2003.

[21] P. Slepička, S. Trostová, N. Slepičková Kasálková et al., "Nanostructuring of polymethylpentene by plasma and heat treatment for improved biocompatibility," Polymer Degradation and Stability, vol. 97, no. 7, pp. 1075-1082, 2012.

[22] V. Švorčík, A. Chaloupka, P. Řezanka et al., "Au-nanoparticles grafted on plasma treated PE," Radiation Physics and Chemistry, vol. 79, no. 3, pp. 315-317, 2010.

[23] V. Švorčík, A. Řezníčková, Z. Kolská, P. Slepička, and V. Hnatowicz, "Variable surface properties of PTFE foils," e-Polymers, vol. 133, no. 1-6, 2010.

[24] A. Vesel and M. Mozetic, "Surface modification and ageing of PMMA polymer by oxygen plasma treatment," Vacuum, vol. 86, no. 6, pp. 634-637, 2012. 

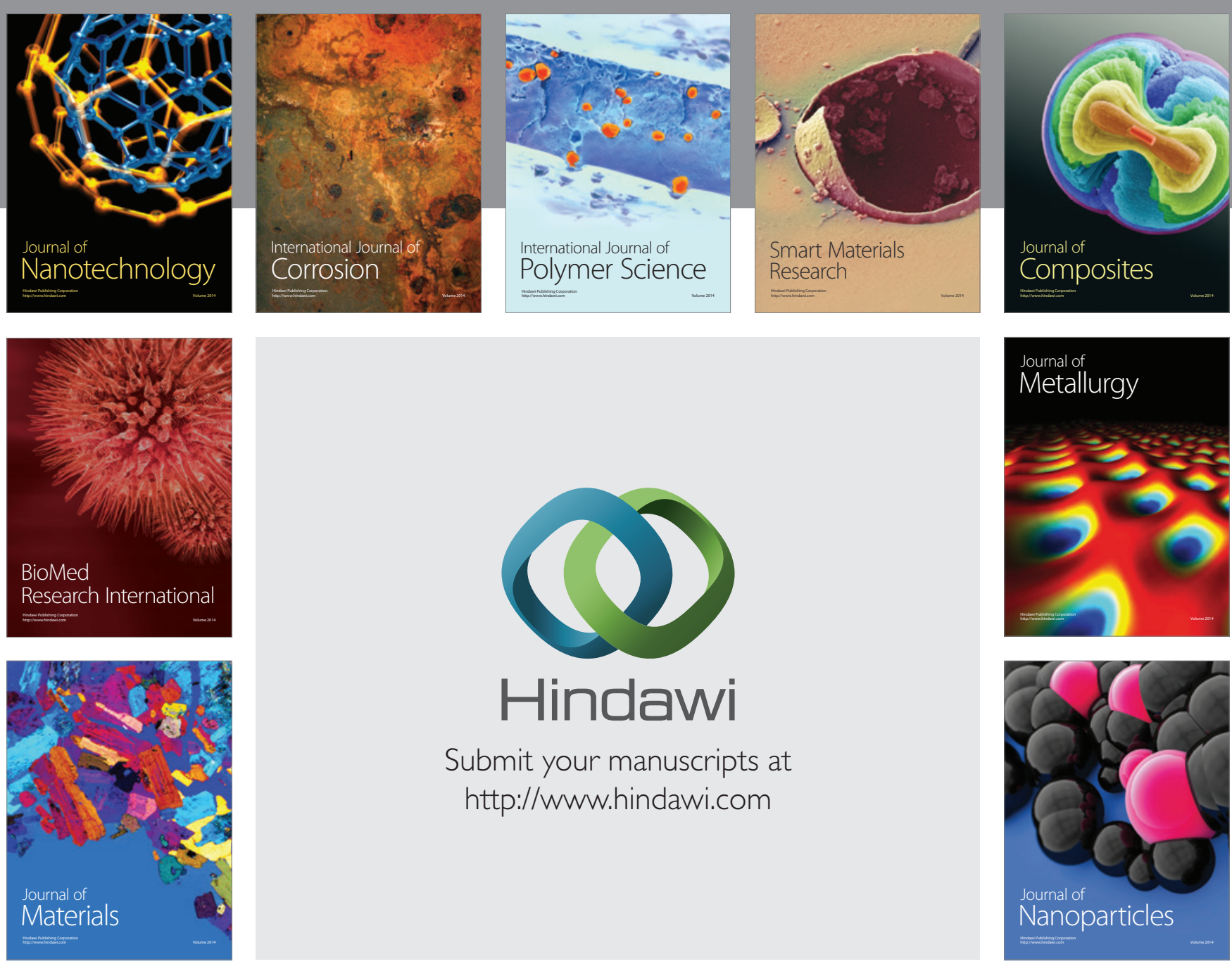

Submit your manuscripts at http://www.hindawi.com
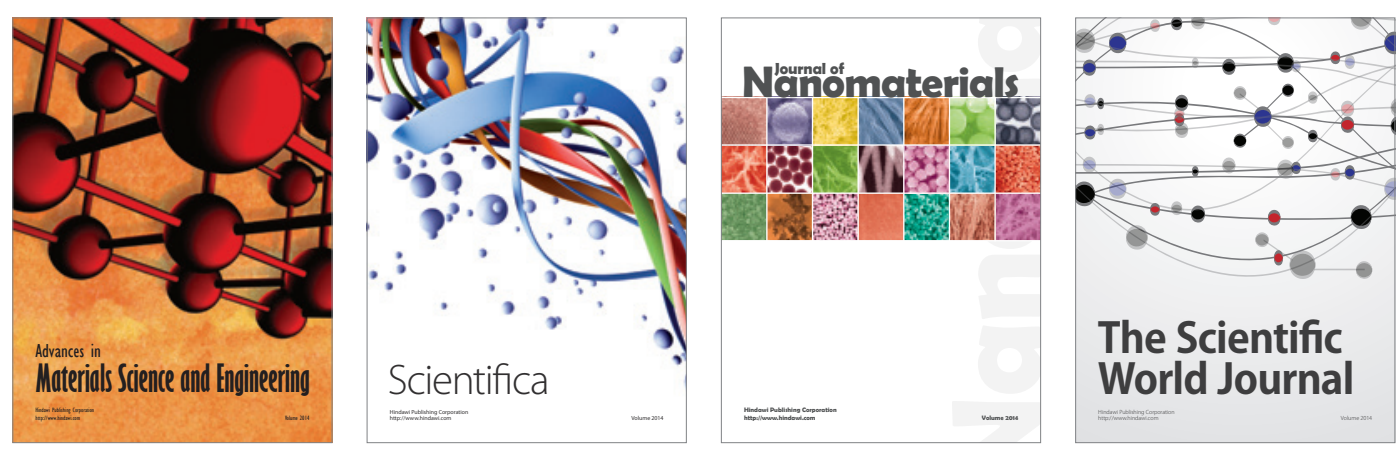

\section{The Scientific World Journal}
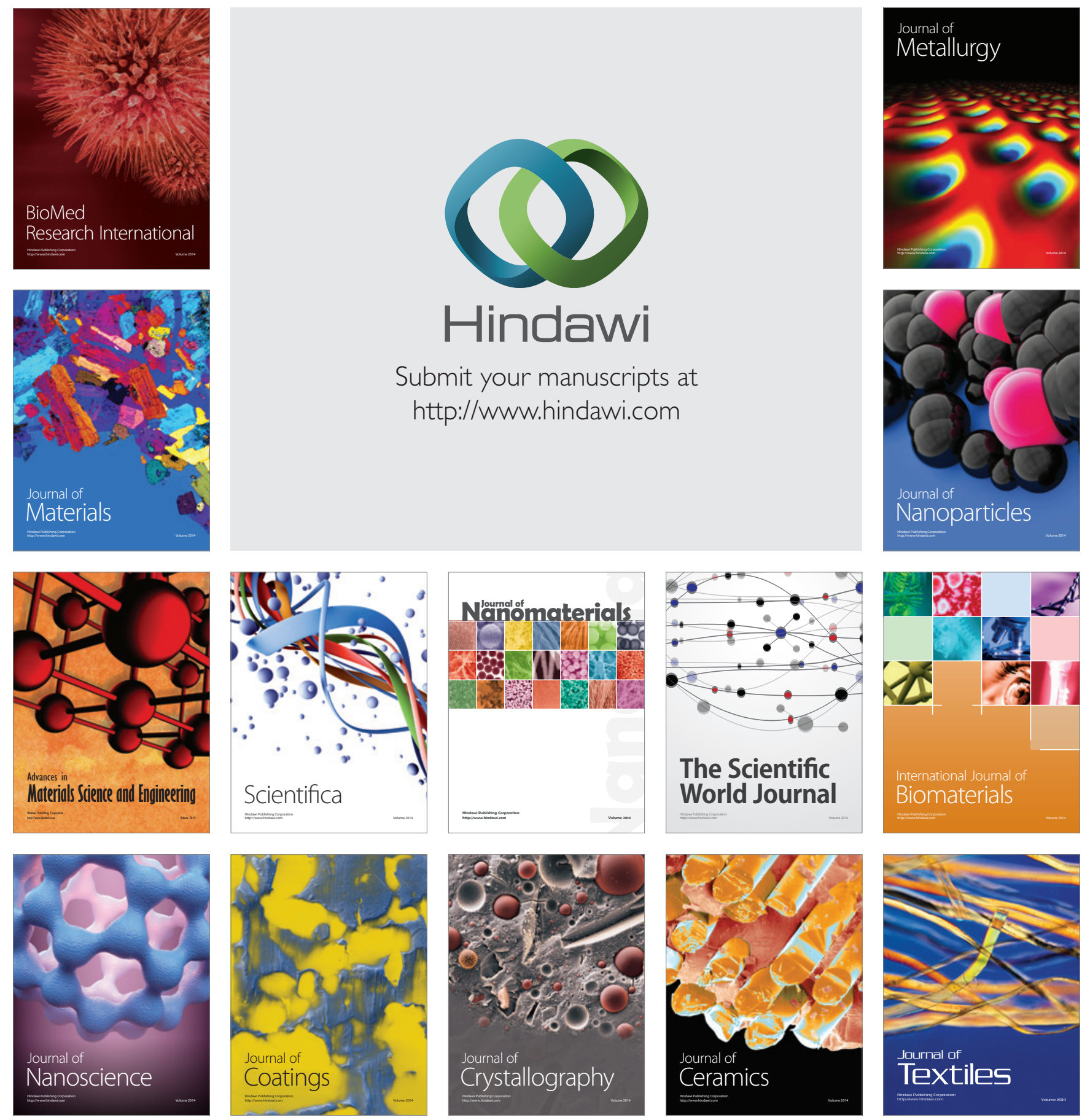